\title{
APPLYING WPD AND SVD TO CLASSIFICATION OF EM WAVE INDUCED BY PARTIAL DISCHARGE IN POWER TRANSFORMER
}

\author{
Xu Zhao* - Yong-Hong Cheng* ${ }^{*}$ \\ Yong-Peng Meng* — Michael G. Danikas ${ }^{* *}$
}

\begin{abstract}
Partial discharge (PD) current is an impulse signal at nanosecond level, which can generate electromagnetic (EM) wave containing broadband frequency information. The frequency band of EM signal is from $\mathrm{MHz}$ up to GHz. Due to different PD patterns, impulse currents with different shapes induce different EM waves containing different frequency information. Therefore, using the features extracted from frequency domain of EM signals, the classification of PD patterns can be effectively got. It is good to use wavelet or wavelet packet decomposition to select features. However, if the decomposition level is too shallow to find enough effective features, it cannot group the EM signals to the right pattern. On the contrary, although it is easier to find features to distinguish the PD pattern if the decomposition level is deep, there will be a lot of redundancy variables and it is hard to select features among so many variables. In this paper, a method is presented, which selected features in the whole decomposition tree instead of selecting among the leaf node of the tree, because more potential features can be found in the whole tree. With the present method, it is possible not only to get enough features, but also to eliminate the redundancy variables effectively. In order to validate the method, large EM signals from four PD patterns in a power transformer are acquired as the training data and testing data for feature selection and classification, and three common classification methods are introduced to classify the PD patterns using the features selected by the method. Most of the classification results are satisfactory indicating that the proposed method is effective.
\end{abstract}

K e y w o r d s: partial discharge, EM signals, classification, wavelet packet, SVD

\section{INTRODUCTION}

Partial discharge (PD) is treated as a main threaten to the insulation system in power equipment. How to detect and identify PD immediately is very important to the safety and operation of power equipment. PD current is an impulse signal at nanosecond level, which can generate electromagnetic (EM) wave containing wide band frequency information. The band width of EM signal is from $\mathrm{MHz}$ up to $\mathrm{GHz}$, so abundant frequency information of PD can be detected using EM measuring technology, which has been widely applied in field to detect PD [1-5].

Because the frequency components of EM signals from different type of PDs are not all the same, it is possible to extract frequency features of EM signals to identify PD patterns. Wavelet or wavelet packet decomposition (WPD) are always treated as the common feature selection method in classification of EM signals. Some of the previous research adopted the information in different frequency band as classification features, which can be got from each tree node in WPD tree, including energy features, fractal dimension features, etc [6-11].

The differences in the shape of PD currents are very small, so the variance of EM signals generated by PDs is minute, the level of WPD should be deep enough to get the features, which can indicate the differences among the EM signals. However, the deeper the decomposition level is, the much larger the number of the energy feature can- didates is. This paper presents a method to do feature selection effectively by WPD and singular value decomposition (SVD). After feature selection, the validity check was used in this paper and the classification results were satisfactory.

In Section 2, WPD and SVD used in this paper are introduced and the definition of node energy is presented. In the third section, it is presented how to select energy features using WPD and SVD. In the fourth section, the experimental system for acquiring EM signals was presented and a large number of EM signals were collected for feature selection and classification using the method introduced in the third section. In the fifth section, three classification models, including support vector machine (SVM), the neural network and the nearest neighbor principle, are introduced to classify PD patterns using energy features of EM signals selected in this paper.

\section{WAVELET PACKET DECOMPOSITION AND SVD}

\subsection{Wavelet Packet Decomposition}

Wavelet decomposition and wavelet packet decomposition (WPD) are the effective tools in analysis of frequency characteristics of transient signals. In order to find the features contained in the EM signals, this paper introduces the wavelet packet decomposition, because the

\footnotetext{
* State Key Laboratory of Power Equipment and Electrical Insulation Xi'an Jiaotong University Xi'an, 710049, China, tuan.zx@stu. xjtu.edu.cn ${ }^{* *}$ Electric Energy Systems Laboratory Department of Electrical and Computer Engineering Democritus University of Thrace Xanthi, Greece, mdanikas@ee.duth.gr
} 


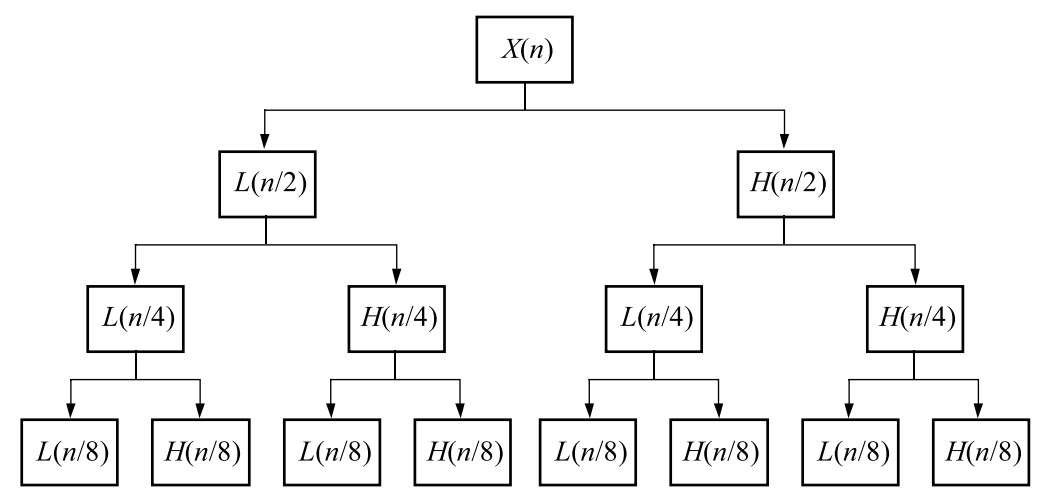

Fig. 1. Sketch of wavelet packet decomposition with three levels

wavelet packet decomposition can do analysis both in the low frequency segment and the high frequency segment for signals, while wavelet decomposition only decompose signals in the low frequency segment and thus loses some details in the high frequency domain. The typical wavelet packet decomposition is shown in Fig. 1.

Each node in WPD tree records the wavelet coefficients, which reflects time-domain characteristics in special frequency band of the signal. Calculating the energy of wavelet coefficients in each node using equation (1), we can get the node energy ex of each node in WPD tree.

$$
e_{x}=\frac{1}{N} x^{\top} x
$$

where $x$ represents the wavelet coefficients of each node, and $N$ is the length of the wavelet coefficients. All the node energies form the node energy vector of the signal, and the energy matrix of a group of EM signals decomposed by wavelet packet is shown in equation

$$
E=\left[\begin{array}{ccccc}
e_{1,1} & e_{1,2} & e_{1,3} & \ldots & e_{1, M} \\
e_{2,1} & e_{2,2} & e_{2,3} & \ldots & e_{2, M} \\
\vdots & \vdots & \vdots & \ddots & \vdots \\
e_{n, 1} & e_{n, 2} & e_{n, 3} & \ldots & e_{n, M}
\end{array}\right]
$$

where $n$ represents the number of nodes and the serial number of nodes in WPD tree is form 1 to $n$. $M$ is the number of EM signals. $e_{i, j}$ represents the $i$ th node energy of the $j$ th EM signal.

\subsection{Singular Value Feature Vector}

The singular value decomposition (SVD) is an effective algebraic feature extraction method. Singular value decomposition technique has been widely applied in the image data compression, signal processing and pattern analysis. A real symmetric matrix can be transformed into a diagonal matrix after orthogonal transformation, and any real matrix $A_{m \times n}$ can be transformed into a diagonal matrix using SVD.

Make $A_{m \times n}$ a real matrix (without loss of generality, set $m \geq n)$ and $\operatorname{rank}(A)=k$, then there exists two orthogonal matrices of $U_{m \times m}$ and $V_{n \times n}$ and the diagonal matrix of $D_{m \times n}$, and the following equation is established [12].

$$
A=U D V^{\top}
$$

Here, $D_{m \times n}=\left(\begin{array}{cc}\Sigma_{k \times k} & 0 \\ 0 & 0\end{array}\right), \Sigma_{k \times k}=\operatorname{diag}\left(\sigma_{1}, \sigma_{2}, \ldots, \sigma_{k}\right)$, $U_{m \times m}^{\top}=\left(u_{1}, u_{2}, \ldots, u_{k}, \ldots, u_{m}\right), V_{n \times n}=\left(v_{1}, v_{2}, \ldots\right.$, $\left.v_{k}, \ldots, v_{n}\right)$, where $\top$ denotes the transpose, $\sigma_{i}=\sqrt{\lambda_{i}}$ $(i=1,2, \ldots, k, \ldots, n)$ are called the singular values of matrix $A . \lambda_{1}, \lambda_{2}, \ldots, \lambda_{k}$ are the whole of nonzero eigenvalues of $A A^{\top}, \lambda_{1} \geq \lambda_{2} \geq \ldots \lambda_{k}>0 . \lambda_{k+1}=\lambda_{k+2}=$ $\cdots=\lambda_{n}=0$ are the $n-k$ zero eigenvalues of $A A^{\top}$. $u_{i}$ and $v_{i}(i=1,2, \ldots, k)$ are the eigenvectors of $A A^{\top}$ and $A^{\top} A$ corresponding to nonzero eigenvalues. Equation (3) can be written in the form

$$
A=\sum_{i=1}^{k} \sigma_{i} u_{i} v_{i}^{\top}
$$

If $A$ represents the energy matrix, equation (4) is the orthogonal decomposition of the energy matrix. Singular values $\sigma_{i}$ in the main diagonal of singular value matrix $\Sigma$, together with the remaining $n-k$ zero elements of $D_{m \times n}$, constitute an $n$-dimensional vector

$$
x_{n \times 1}=D_{n \times n} e=\left(\sigma_{1}, \ldots, \sigma_{k}, 0, \ldots, 0\right)^{\top}
$$

where $D_{n \times n}$ is an $n$-order sub-vector in $D, e=(1,1$, $\ldots, 1)^{\top}$, and $x_{n \times 1}$ is called the singular value eigenvector of A. For any real matrix, up to the conditions $\lambda_{1} \geq$ $\lambda_{2} \geq \cdots \geq \lambda_{k}$, singular value diagonal matrix is unique. Therefore, one energy matrix E corresponds to one unique singular value feature vector.

\section{FEATURES SELECTION FOR EM SIGNALS}

In [6-9] it was presented that using wavelet or wavelet packet decomposition extraction of energy features and the classification results were satisfactory, while the methods mentioned in these papers focused on selecting energy features among the leaf nodes in WPD tree. In this paper, according to the analysis of WPD of EM signals, it 

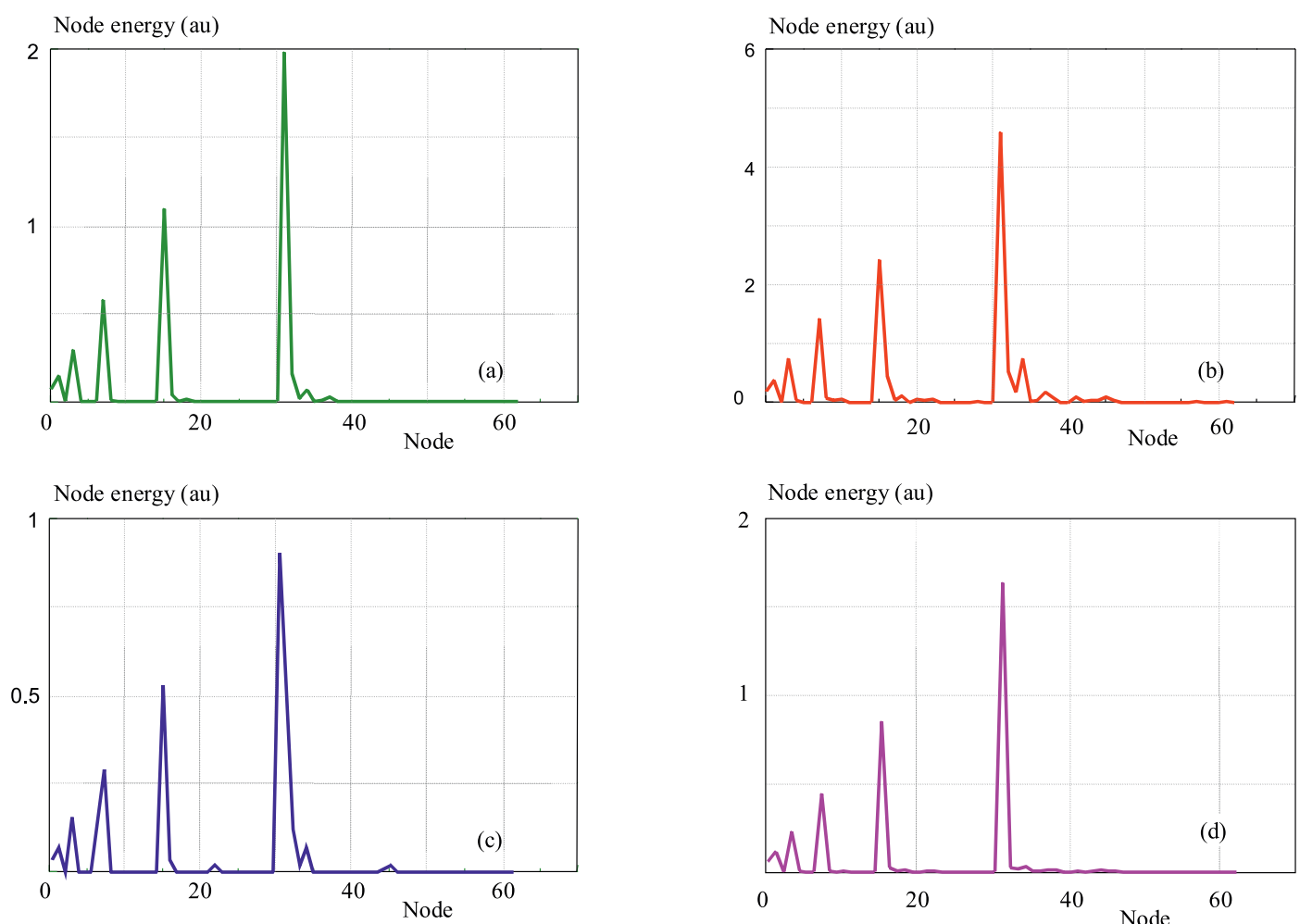

Fig. 2. Energy vector of EM signals induced by four type of PD pattern using wavelet packet decomposition, depth=5, mother wavelet ='db8': (a) - floating, (b) - needle, (c) - oil clearance, (d) - void
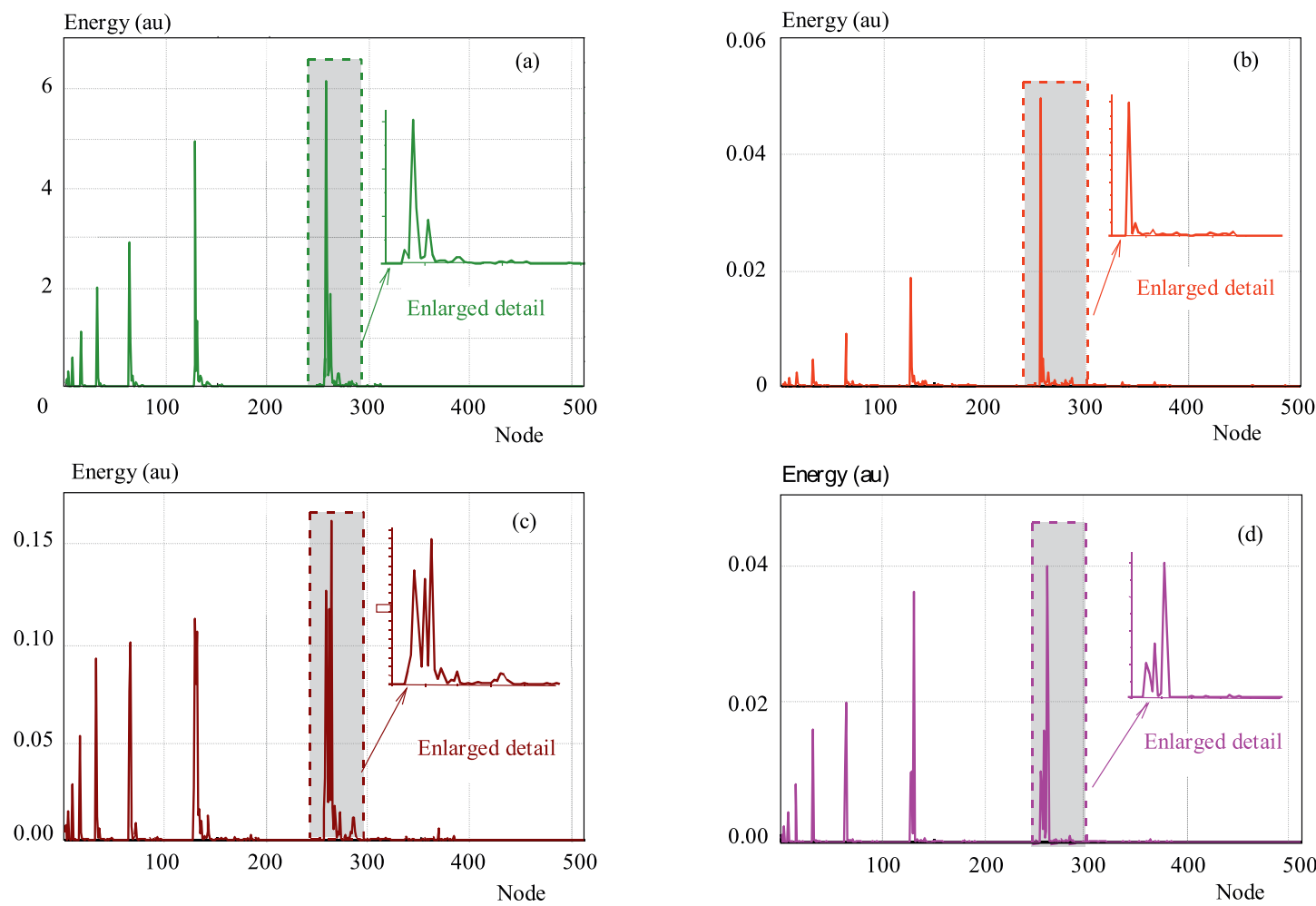

Fig. 3. Energy vector of EM signals induced by four type of PD pattern using wavelet packet decomposition, depth=8, mother wavelet ='db8': (a) - floating, (b) - needle, (c) - oil clearance, (d) - void

is found that, if the level of decomposition is not deep enough, there would not be effective parameters for classification. In other words, the PD pattern cannot be classified using these parameters. Figure 2 shows the energy vectors of EM signals from four types of PD patterns.
The decomposition depth is five and the mother wavelet is 'db8'. There are almost no differences among the energy vectors except the amplitude. So that, it cannot be classification of PD pattern using energy vectors of EM signals with this depth of WPD. Figure 3 shows the en- 


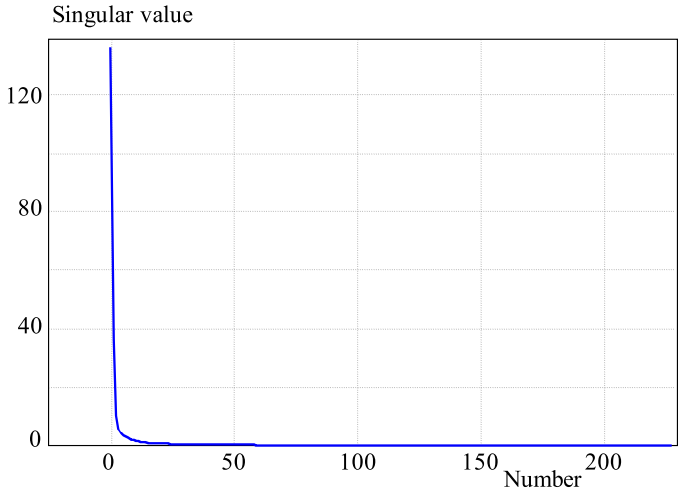

Fig. 4. Singular value of the needle-plane energy vector matrix $E$

ergy vectors of EM signals, the depth of WPD is eight and the mother wavelet is 'db8'. The enlarge detail of energy vector in the $8^{\text {th }}$ level is also shown in Fig. 3. It is obvious that, if the depth of WPD is deep enough, the parameters from the energy vector can be selected as energy features for classification of PD patterns.

However, if the depth of WPD is too deep, the number of tree node must increase geometrically. Taking the $8^{\text {th }}$ level of WPD as an example, the number of tree node is up to 511 . To be able to extract energy features from these energy vectors, the paper introduced SVD mentioned in
Section 1 to select energy features. The step of feature selection is as follows:

1) doing 8 level WPD for EM signals;

2) calculating energy of each node in decomposition tree for each EM signal, composing the energy vector of each EM signal;

3) constructing the energy matrix $E$ using all the energy vector of EM signals from the same PD pattern;

4) calculating the singular value of each $E$ of different type of PD patterns shown in Fig. 4;

5) obtaining primary singular vectors depending on the singular value for each kind of EM signals shown in Fig. 5a and b;

6) selecting a collection of nodes from the WPD tree nodes depending on their component in the primary singular vectors shown in Fig. 5a and b;

Finding the collection should obey the following principle. First, the collection is constructed with the node if its component is bigger than $5 \%$ of the maximum component of that primary singular vector shown in Fig. 5. Second, reduction of the collection is desirable for elimination of superfluous information. An energy feature group which contains parent node energy and all of its descendant node energy may be redundant because any parent node of WPD tree can be constructed from its children
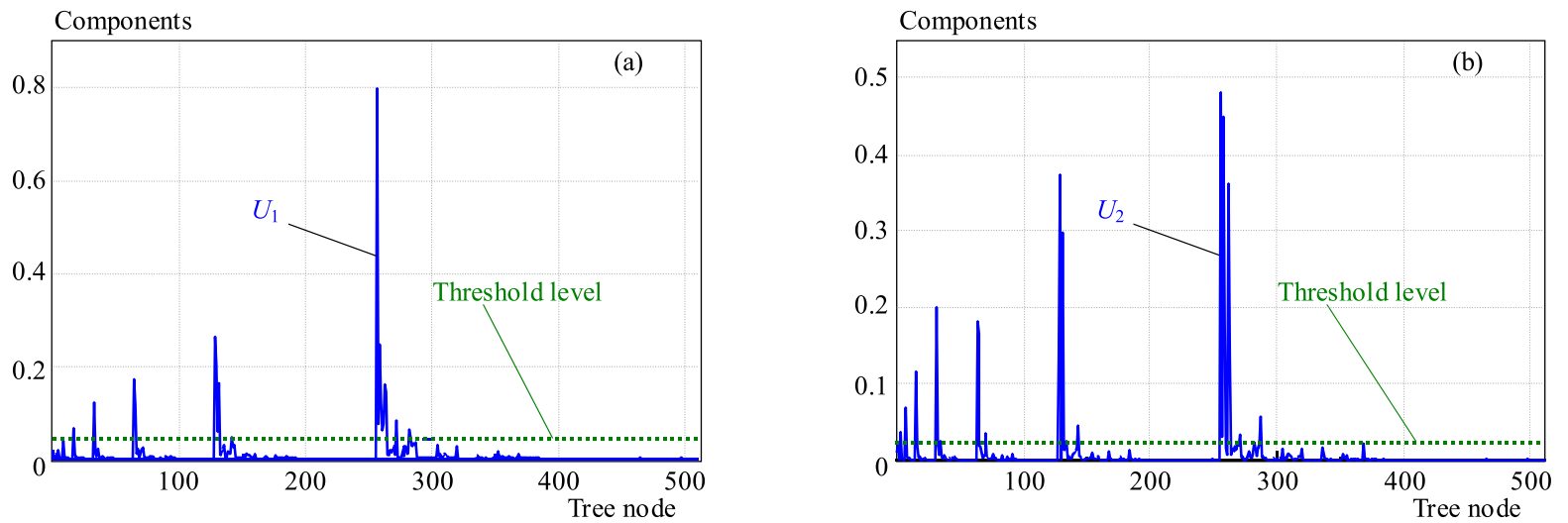

Fig. 5. Singular vectors of energy matrix E of needle: (a) - singular vector corresponding to largest singular value (b) - singular vector corresponding to the second largest singular vector

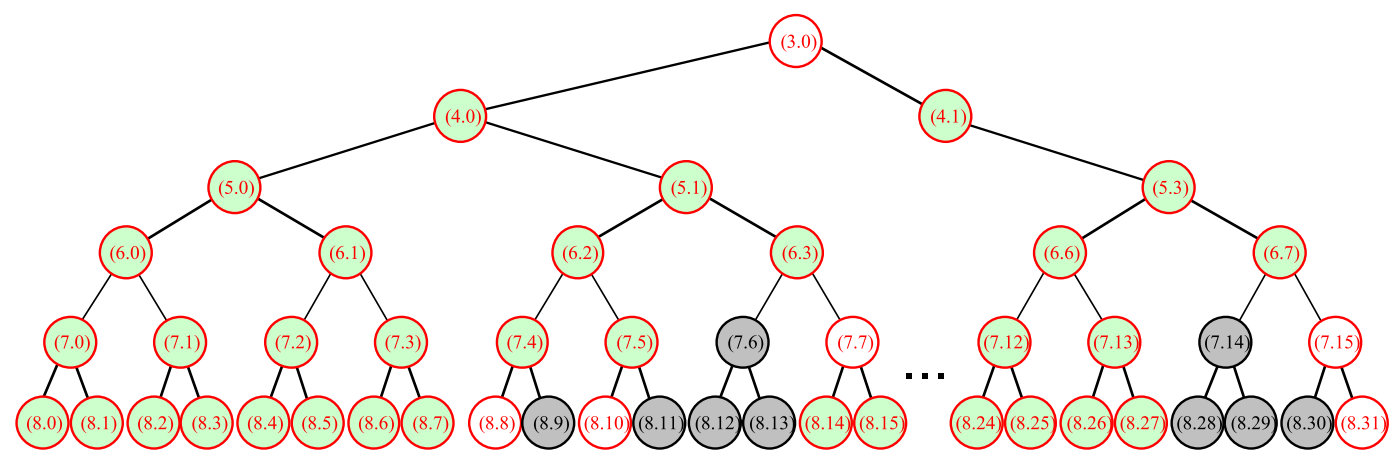

Fig. 6. The scheme of reduction of the collection 

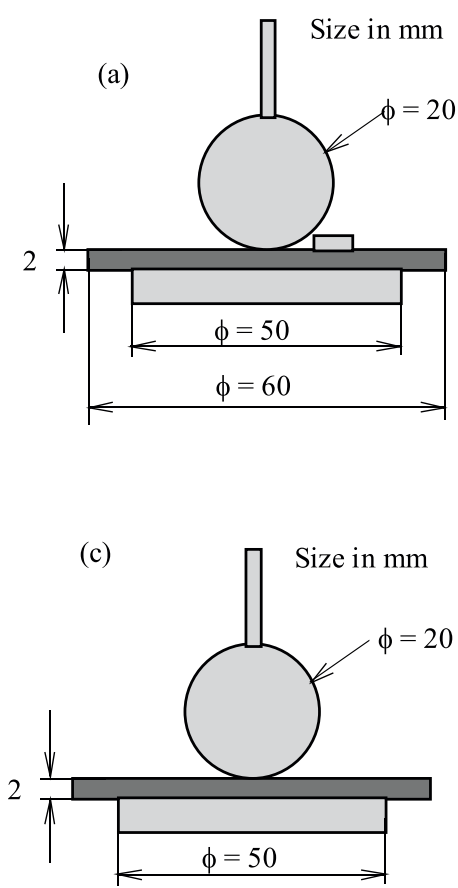
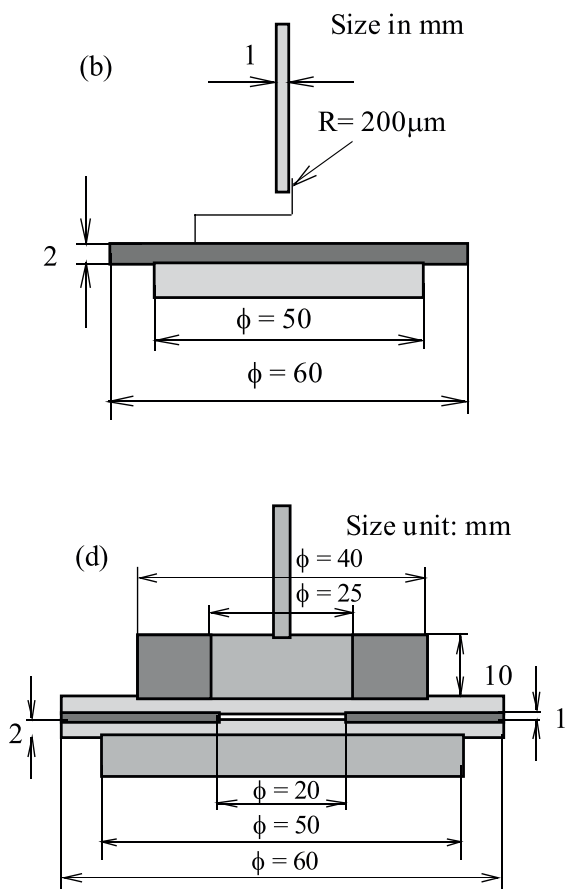

Fig. 7. Classic PD pattern in power transformer: (a) - floating (b) - needle (c) - oil clearance (d) - void in press-board

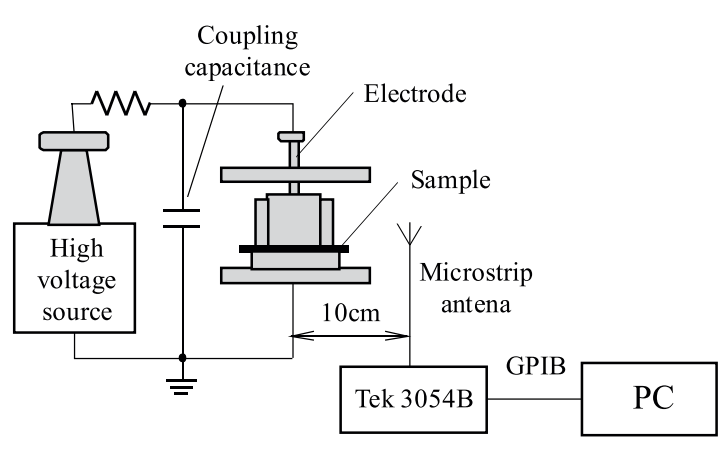

Fig. 8. The experiment setup

nodes. Therefore, an energy feature group is appropriate if the group does not contain both parent and all of the children energy nodes within a given singular value. In that case, the parent node should be reserved as an energy feature while all of its children nodes should be discarded.It must be notified that, if both the parent and one of its children nodes are in the collection, each of them should be preserved as energy feature. This is because the child node contains some detail information. The scheme of reduction of the collection is shown in Fig. 6. Finally, the collection is the energy feature set. Step 1 to step 3 have been mentioned above, the detailed information of step 4 to step 6 is as follows.

In Section 2, it is mentioned that the bigger the singular value is, the more various the energy vector is in new variable space. In this paper, energy vector matrix $E$ of EM signals from four types of PD patterns were obtained; the singular values of different $\mathrm{E}$ were calculated shown in Table 1. Figure 4 shows the singular value of energy vector matrix of EM signals from the pattern of needle-plane PD.
In Fig. 4, the singular values of energy vector decrease sharply, which contains only two primary singular values, that means there are two new main energy vectors contributing to the main change in new variable space. The singular values of the energy vector $E$ from four types of PD patterns contain 1 or 2 primary singular values; the ratio between singular values is used to express the variation among singular values

$$
\begin{aligned}
& \Delta \delta_{12}=\frac{\delta_{1}-\delta_{2}}{\delta_{1}}, \\
& \Delta \delta_{23}=\frac{\delta_{2}-\delta_{3}}{\delta_{2}}, \\
& \Delta \delta_{34}=\frac{\delta_{3}-\delta_{4}}{\delta_{3}}
\end{aligned}
$$

where $\delta_{1}, \delta_{2}, \delta_{3}$ and $\delta_{4}$ are the biggest four singular values of, $\Delta \delta_{12}, \Delta \delta_{23}$ and $\Delta \delta_{34}$ are the ratios between two singular values. Table 1 shows the $\Delta \delta_{12}, \Delta \delta_{23}$ and $\Delta \delta_{34}$ of four types of PD patterns. It can be seen that, conducted by SVD, $E$ of floating PD, oil clearance PD and void $\mathrm{PD}$ in pressboard is constructed with a primary singular vector, while $E$ of needle PD is constructed with two primary singular vectors. After executing these steps above, seven energy features are finally extracted, which are $(8,31),(8,10),(8,8),(7,7),(7,13),(5,3),(3,0)$. The first digit in bracket denotes the level of WPD; the second digit denotes the position of tree node in the level indicated by the first digit. The result of feature selection is shown in Fig. 6, where all the white dots are the nodes selected after the step 6 was finished, the dots without deletion line in white nodes are the final features obeying the selecting principle mentioned above, while the white nodes with deletion line are discarded nodes. Other gray nodes are just redundant nodes. 

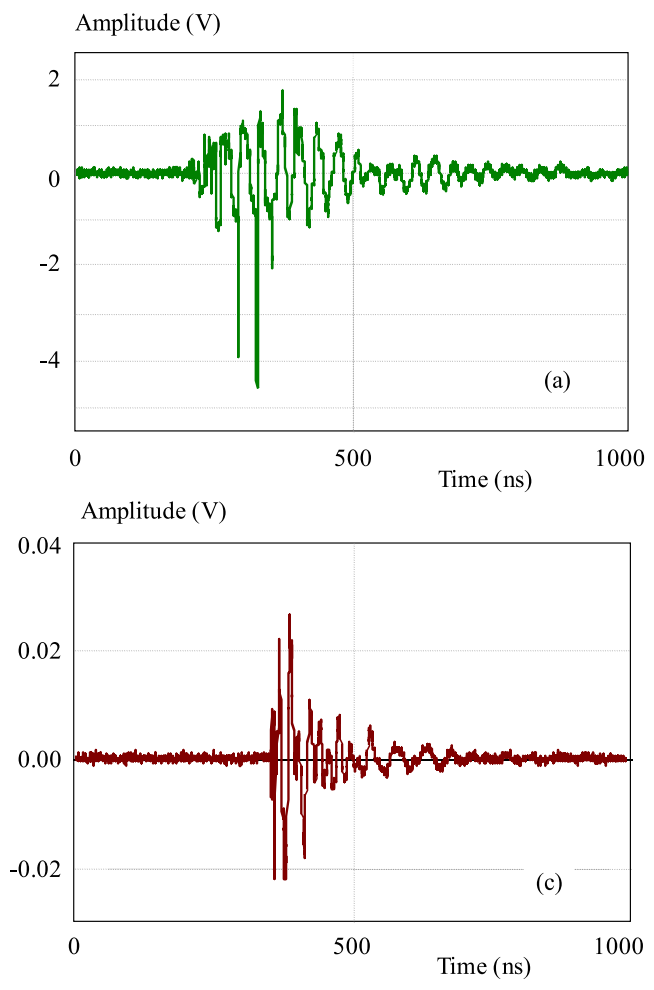
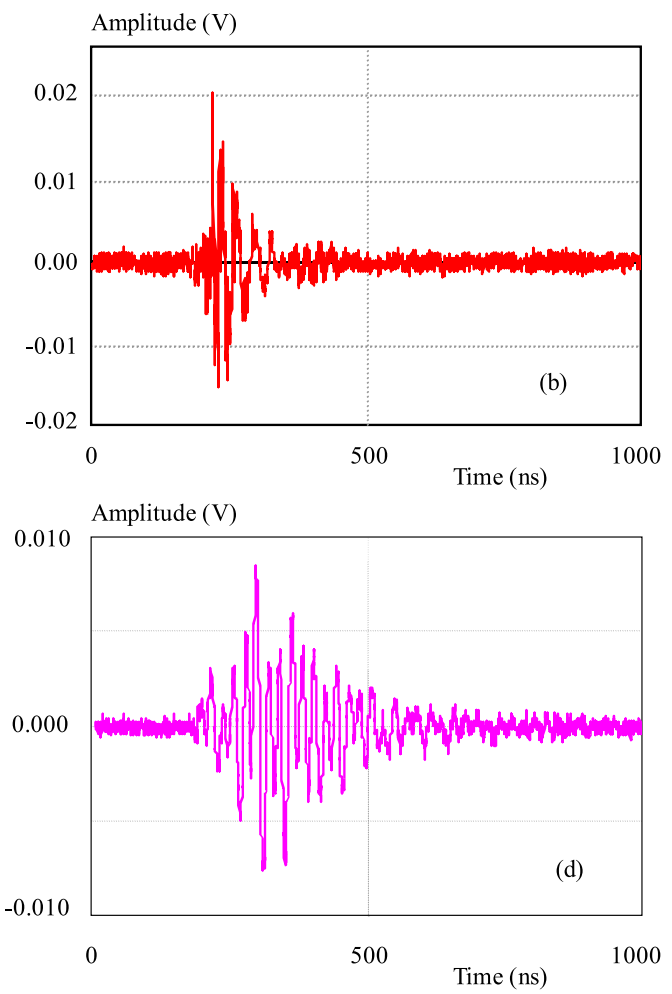

Fig. 9. Classic EM signals: (a) - floating (b) - needle (c) - oil clearance (d) - void in press-board

\section{SAMPLES AND EXPERIMENT}

\subsection{Experimental Setup}

For validity checking of the method presented in this paper, four classic PD patterns, including floating PD, needle PD, oil clearance PD and void PD in press-board, were prepared (shown in Fig. 7). More than thousands of EM signals induced by the four types of PD patterns were collected for checking the availability of this method. The scheme of experimental setup is shown in Fig. 8. The EM sensor is a micro-strip antenna. The oscilloscope is Tek3054B, the sample frequency is $5 \mathrm{GS} / \mathrm{s}$ and the band width is $500 \mathrm{MHz}$. Furthermore, because the frequency characteristic of EM signals has a relationship with the propagation distance [15], the distance between the sensor and the PD source is $10 \mathrm{~cm}$. The model of four classic types of PD patterns is shown in Fig. 8. In the model of floating PD, an equilateral triangle with the length of side $5 \mathrm{~mm}$ is treated as the floating electric potential. The classic EM signals generated by the four PD patterns are shown in Fig. 9. Table 3 shows the number of samples from the four types of PD pattern and the measuring voltage.

\subsection{Feature Selection For Sample Data}

Ternary diagram is a very useful and intuition diagram to show the distribution of data corresponding to three parameters.In this section, using the energy features selected in Section 2, the EM signals from four types of
PD patterns can be classified directly in ternary diagram (Fig. 10), where it is obviously that the gravity centers of distribution of four types of EM signals, around with circle or ellipse, are separated. Feature 1 denotes $(3,0)$, feature 2 denotes $(5,3)$ and feature 3 denotes $(8,10)$.

\section{VALIDITY CHECKING OF ENERGY FEATURES}

In this section, three common classification model, including support vector machine (SVM), Radial basis function (RBF) neutral network and the nearest neighbor principle, are introduced for validity checking using features selected by the method presented in this paper.

Due to the different energy level of PD patterns, the energies of EM signals are at a different level, for example, the energy vector of EM signals from needle is much larger than the others (Fig. 3). Therefore, normalization should be conducted to eliminate the interference of energy level

$$
e=\left(e_{x}-\operatorname{mean}\left(e_{x}\right)\right) / \operatorname{std}\left(e_{x}\right)
$$

where $e_{x}$ is the energy feature vector, mean $\left(e_{x}\right)$ is the mean value of the vector, $\operatorname{std}\left(e_{x}\right)$ is the standard deviation of the energy feature vector

Using LIBSVE [13] to train and test the samples, radial bases function (RBF) was chosen to be the base function

$$
\exp \left(-\gamma|u-v|^{2}\right)
$$




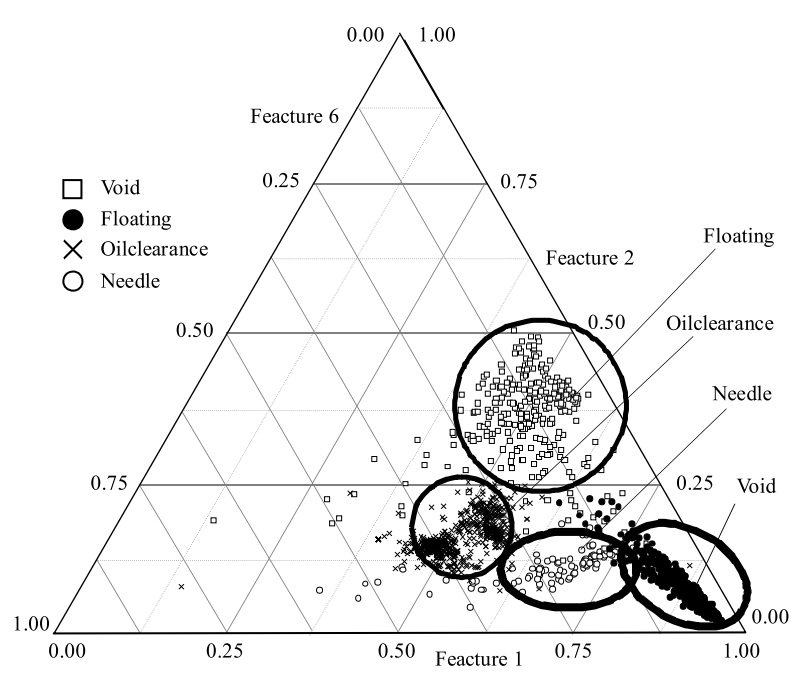

Fig. 10. The ternary diagram of the four types of PD patterns

Table 1. Singular values of $E$ from four types of PD patterns

\begin{tabular}{ccccc}
\hline PD pattern & $\delta_{1}$ & $\delta_{2}$ & $\delta_{3}$ & $\delta_{4}$ \\
\hline Floating & 137 & 36 & 10 & 5 \\
Needle & 0.0077 & 0.0056 & $9.2 \times 10^{-4}$ & $5.6 \times 10^{-4}$ \\
Oil clearance & 0.006 & 0.0017 & $4.6 \times 10^{-4}$ & $3.3 \times 10^{-4}$ \\
Void in pressboard & 0.0016 & $4.4 \times 10^{-4}$ & $3.8 \times 10^{-4}$ & $1.7 \times 10^{-4}$ \\
\hline
\end{tabular}

Table 2. The ratio of singular values from four type of pd patterns

\begin{tabular}{cccc}
\hline PD pattern & $\Delta \delta_{12}$ & $\Delta \delta_{23}$ & $\Delta \delta_{34}$ \\
\hline Floating & 0.74 & 0.72 & 0.5 \\
Needle & 0.27 & 0.84 & 0.39 \\
Oil clearance & 0.72 & 0.73 & 0.28 \\
Void in pressboard & 0.73 & 0.14 & 0.55 \\
\hline
\end{tabular}

where all the parameters in (5) are the default value. gamma is $1 / 7$, where 7 is the number of energy features. Penalty factor $C$ is 1 .

All the classification results are shown in the Tables 47. The classification result using SVM is very satisfactory (Tab. 4). Table 5 shows the classification result using RBF neutral network with the Mean Squared Error (MSE) 0.05 and 75 neurons, while Table 6 shows the result from RBF neutral network with the MSE 0.01 and 350 neurons. It can be seen from the classification results that there is an over fitting with the MSE 0.01 and 350 neurons. Although the number of neurons is more, the accuracy of classification is lower. This means that for RBF neutral network, a high number of neurons may not lead to good accuracy of classification.

It also can be seen from the classification result that the classification accuracy of SVM and nearest neighbor principle is excellent, while the classification result of RBF neutral network for floating PD and needle PD is not very satisfactory (Tab. 6).
As a conclusion, one may say that although any of parameters was not optimized for the three classification model, the classification results were very good. Therefore, the energy features selected by the method of this paper are very effective for classification of PD patterns.

Table 3. Measuring voltage and the number of samples of EM signals from the four type PD patterns

\begin{tabular}{ccc}
\hline PD pattern & $\begin{array}{c}\text { Measuring voltage } \\
(\mathrm{kV})\end{array}$ & $\begin{array}{c}\text { Number } \\
\text { of sample }\end{array}$ \\
\hline Floating & 20 & 229 \\
Needle & 27 & 301 \\
Oil clearance & 22 & 599 \\
Void in pressboard & 7.5 & 428 \\
\hline
\end{tabular}

Table 4. Classification result of SVM

\begin{tabular}{ccc}
\hline PD pattern & $\begin{array}{c}\text { Classification result } \\
\text { correct number/test number }\end{array}$ & $\begin{array}{c}\text { Accuracy } \\
(\%)\end{array}$ \\
\hline Floating & $49 / 50$ & 98 \\
Needle & $58 / 60$ & 96.7 \\
Oil clearance & $118 / 120$ & 98.3 \\
Void in pressboard & $80 / 80$ & 100 \\
\hline
\end{tabular}

Table 5. Classification result of RBF neutral network MSE 0.05 and neurons 75

\begin{tabular}{ccc}
\hline \multirow{2}{*}{ PD pattern } & $\begin{array}{c}\text { Classification result } \\
\text { correct number/test number }\end{array}$ & $\begin{array}{c}\text { Accuracy } \\
(\%)\end{array}$ \\
\hline Floating & $42 / 50$ & 84 \\
Needle & $58 / 60$ & 96.7 \\
Oil clearance & $117 / 120$ & 97.5 \\
Void in pressboard & $79 / 80$ & 98.75 \\
\hline
\end{tabular}

Table 6. Classification result of RBF neutral network MSE 0.01 and neurons 350

\begin{tabular}{ccc}
\hline PD pattern & $\begin{array}{c}\text { Classification result } \\
\text { correct number/test number }\end{array}$ & $\begin{array}{c}\text { Accuracy } \\
(\%)\end{array}$ \\
\hline Floating & $43 / 50$ & 86 \\
Needle & $52 / 60$ & 86.7 \\
Oil clearance & $115 / 120$ & 95.8 \\
Void in pressboard & $80 / 80$ & 100 \\
\hline
\end{tabular}

Table 7. Classification result of nearest neighbor principle

\begin{tabular}{ccc}
\hline PD pattern & $\begin{array}{c}\text { Classification result } \\
\text { correct number/test number }\end{array}$ & $\begin{array}{c}\text { Accuracy } \\
(\%)\end{array}$ \\
\hline Floating & $48 / 50$ & 96 \\
Needle & $59 / 60$ & 98.3 \\
Oil clearance & $119 / 120$ & 99.2 \\
Void in pressboard & $80 / 80$ & 100 \\
\hline
\end{tabular}




\section{CONCLUSION}

In this paper, a new method of feature selection of EM signals was presented, the energy vector of EM signals was analyzed using WPD and the energy features in different frequency band were selected using SVD. Then, different types of $\mathrm{PD}$ patterns in a power transformer were identified using energy features selected in this paper. Moreover, the depth of WPD is a main factor of feature selection. If the depth is shallow, there will not effective variables for classification, at the same time, if the depth is deep enough there will be too many variables selected. So that, in Section 2, the energy of each node in the WPD tree was treated as the energy feature candidate, and the depth of WPD should be deep enough for feature selection, the depth is eight in this paper. Due to the large number of energy feature candidates, SVD was introduced to extract energy features. After the processing of SVD, the redundancy among the feature candidates should be eliminated. Finally, seven energy features were selected for classification. In Section 4, three common classification models were introduced to check the availability of energy features and the results were very satisfactory.

\section{REFERENCES}

[1] JUDD, M. D.-LI YANG-HUNTER, I. B. B.: Partial Discharge Monitoring for Power Transformers Using EM Sensors Part 1: Sensors and Signal Interpretation, IEEE Electrical Insulation Magazine 21 No. 2 (2005), 5-14.

[2] JUDD, M. D.-LI YANG-HUNTER, I. B. B.: Partial Discharge Monitoring for Power Transformers Using EM Sensors Part 2: Field Experience, IEEE Electrical Insulation Magazine 21 No. 3 (2005), 5-13.

[3] ZHAO, X.-CHENG, Y.-MENG, Y.: Development and Application of Integrated Multi-Parameter On-Line Monitoring System for Power Transformer, J. Electric Power 44 No. 7 (2011).

[4] JUDD, M. D.-HAMPTON, B. F.-BROWN, W. L.: EM Partial Discharge Monitoring for $132 \mathrm{kV}$ GIS, Proc. 10th International Symposium on High Voltage Engineering, August 1997, Montral, Canada.

[5] JUDD, M. D.: Experience with EM Partial Discharge Detection and Location in Power Transformers, Electrical Insulation Conference (EIC), Annapolis, MD, USA, 2011, pp. 201-205.

[6] TANG, J.-ZHOU, J. B.-ZHANG, X. X.-TAO, J. G.: Feature Extraction Method Based on Pulse-Coupled Neural Networks for Ultra High Frequency Partial Discharge in Transformers, International Review Of Electrical Engineering (IREE) 7 No. 1 (Jan-Feb 2012).

[7] UMAMAHESWARI, R.-SARATHI, R. : Identification of Partial Discharges in Gas-insulated Switchgear by Ultra-High-Frequency Technique and Classification by Adopting Multi-class Support Vector Machines, Electric Power Components and Systems 39 No. 14 (2011), 1577-1595.

[8] XIE YANBIN—TANG, J.-ZHOU, Q. : Feature Extraction and Recognition of EM Partial Discharge Signals in GIS Based on Dual-Tree Complex Wavelet Transform, European Transactions on Electrical Power 20 No. 5 (2010), 639-649.
[9] Chang, C. S.-LEE, K. H.-CHANG, C.: Recognition of Partial Discharge Waveshape Patterns for Gas Insulated Switchgear, Australasian Universities Power Engineering Conference (AUPEC 2004), 26-29 September 2004, Brisbane, Australia.

10] LI LiXUE-HUANG, C.-ZENG, Y.-JIANG, X. : Partial Discharge Diagnosis on GIS Based on Envelope Detection, WSEAS Transactions on Systems 7 No. 11 (2008), 1238-1247.

[11] RUDD, S.-McARThUR, S. D. J.-JUDD, M. D.: A Generic Knowledge-based Approach to the Analysis of Partial Discharge Data, IEEE Transactions on Dielectrics and Electrical Insulation 17 No. 1 (2010), 149-156.

[12] GOLUB, G. H.-KAHAN, W. : Calculating the Singular Values and Pseudo-Inverse of a Matrix, Journal of the Society for Industrial and Applied Mathematics: Series B, Numerical Analysis 2 No. 2, 205-224.

13] CHIH-CHUNG CHANG-CHIH-JEN LIN : LIBSVM: a Library for Support Vector Machines, ACM Transactions on Intelligent Systems and Technology (2011), 2:27:1-27:27, Software available at http://www.csie.ntu.edu.tw/ ${ }^{\sim}$ cjlin/libsvm.

14] ShibuYA, Y.-MATSUmoto, S.-TANAKA, M.-MUTO, H.-KANEDA, Y.: Electromagnetic Waves from Partial Discharges and their Detection Using Patch Antenna, IEEE Transactions on Dielectrics and Electrical Insulation 17 No. 3 (2010), 862-871.

Received 24 September 2012

Xu Zhao is a $\mathrm{PhD}$ candidate at the Xian Jiaotong University, State Key Laboratory for Electrical Insulation and Power Equipment. His research interests include diagnostic techniques in transformer insulation as well as study of partial discharges in conventional polymers and in nanocomposite polymers.

Yong-Hong Cheng was born in Anhui, China in 1965. He received the MS and $\mathrm{PhD}$ degrees in electrical engineering from Xi'an Jiaotong University, China in 1991 and 1999, respectively. Since 2001, he is a Professor of Xian Jiaotong University, State Key Laboratory of Electrical Insulation and Power Equipment (SKLEIPE).

Yong-Hong Meng is lecturer at Xian Jiaotong University. His research interests are in partial discharges, transformer insulation diagnostics and simulation of pre-breakdown phenomena in both solid and liquid insulation.

Michael G. Danikas received his BSc and MSc degrees from the Dept. of Electrical and Electronic Engineering, University of Newcastle-upon-Tyne, England, in 1980 and 1982, respectively. He received his $\mathrm{PhD}$ from the Dept. of Electrical and Electronic Engineering, Queen Mary College, University of London, England, in 1985. He is currently Professor at Democritus University of Thrace, Dept. of Electrical and Computer Engineering, Xanthi, Greece. During the summer of 2001 he was Visiting Professor in Helsinki University of Technology, High Voltage Laboratory and during the academic year 20082009 he was a Visiting Scholar at Waseda University, Graduate School of Information, Production and Systems. He cooperates with Xi;an Jiaotong University and he spent some time during the summer of 2010 and during the autumn of 2011 working with the State Key Laboratory of Electrical Insulation and Power Equipment (SKLEIPE). 